\title{
Multiple Xanthomonas euvesicatoria Type III Effectors Inhibit flg22-Triggered Immunity
}

\author{
Georgy Popov, ${ }^{1}$ Malou Fraiture, ${ }^{2}$ Frederic Brunner, ${ }^{2}$ and Guido Sessa ${ }^{1}$ \\ ${ }^{1}$ Department of Molecular Biology and Ecology of Plants, Tel-Aviv University, 69978 Tel-Aviv, Israel; and ${ }^{2}$ Department of \\ Biochemistry, Centre for Plant Molecular Biology, Eberhard Karls University, Auf der Morgenstelle 32, D-72076 Tübingen, \\ Germany
}

Accepted 18 July 2016.

\begin{abstract}
Xanthomonas euvesicatoria is the causal agent of bacterial spot disease in pepper and tomato. $X$. euvesicatoria bacteria interfere with plant cellular processes by injecting effector proteins into host cells through the type III secretion (T3S) system. About 35 T3S effectors have been identified in $X$. euvesicatoria 85-10, and a few of them were implicated in suppression of pattern-triggered immunity (PTI). We used an Arabidopsis thaliana pathogen-free protoplast-based assay to identify $X$. euvesicatoria 85-10 effectors that interfere with PTI signaling induced by the bacterial peptide flg22. Of 33 tested effectors, 17 inhibited activation of a PTI-inducible promoter. Among them, nine effectors also interfered with activation of an abscisic acid-inducible promoter. However, effectors that inhibited flg22-induced signaling did not affect phosphorylation of mitogen-activated protein (MAP) kinases acting downstream of flg22 perception. Further investigation of selected effectors revealed that XopAJ, XopE2, and XopF2 inhibited activation of a PTI-inducible promoter by the bacterial peptide elf18 in Arabidopsis protoplasts and by flg22 in tomato protoplasts. The effectors XopF2, XopE2, XopAP, XopAE, ХopH, and XopAJ inhibited flg22-induced callose deposition in planta and enhanced disease symptoms caused by attenuated Pseudomonas syringae bacteria. Finally, selected effectors were found to localize to various plant subcellular compartments. These results indicate that $X$. euvesicatoria bacteria utilize multiple T3S effectors to suppress flg22-induced signaling acting downstream or in parallel to MAP kinase cascades and suggest they act through different molecular mechanisms.
\end{abstract}

Plants utilize a complex detection and signaling network for early recognition of invading microbes and activation of immune responses (Jones and Dangl 2006). Pathogen recognition receptors (PRRs) localized on the external surface of the plant cell detect conserved pathogen-associated molecular patterns (PAMPs), such as fungal chitin or bacterial flagellin, elongation factor-Tu, lipopolysaccharides, and peptidoglycan (Macho and Zipfel 2014). Among the best characterized PRRs is the flagellin-sensing 2 (FLS2) receptor from Arabidopsis thaliana, a receptor like-kinase (RLK) with an extracellular leucine-rich domain (Gómez-Gómez and Boller 2000). FLS2 recognizes bacterial flagellin or its 22-amino acid peptide derivative flg22

Corresponding author: G. Sessa; E-mail: guidos@post.tau.ac.il; Telephone: +1.972.3.640.9766; Fax: +1.972.3.640.9380.

*The $\boldsymbol{e}$-Xtra logo stands for "electronic extra" and indicates that five supplementary figures and one supplementary table are published online.

(c) 2016 The American Phytopathological Society in many plant species, including Arabidopsis, tomato, tobacco, and potato, but not in rice (Felix et al. 1999). PRRs are part of multiprotein complexes that include the receptor, coreceptors, regulatory proteins, and molecular components involved in intracellular signaling (Böhm et al. 2014; Macho and Zipfel 2014). Recognition of PAMPs by PRR complexes often results in phosphorylation and activation of receptor-like cytoplasmic kinases (RLCK) (Lin et al. 2013; Macho and Zipfel 2014). Downstream cellular events include ion fluxes, activation of mitogen-activated protein (MAP) kinase cascades and calciumdependent protein kinases, production and release of reactive oxygen species, callose deposition at the cell wall, and accumulation of pathogenesis-related proteins (Boller and Felix 2009). Collectively, these immune responses are referred to as patterntriggered immunity (PTI) and mediate plant resistance against a broad spectrum of microbial pathogens (Zhang and Zhou 2010).

A prerequisite for plant pathogens to colonize their host is the suppression of PTI. Many phytopathogenic gram-negative bacteria achieve this by injecting effector proteins into the plant cell through the type III secretion (T3S) system (Galán et al. 2014). Once inside the plant cell, T3S effectors manipulate important hubs of immune signaling making the host vulnerable to infection (Feng and Zhou 2012; Macho and Zipfel 2015). Several T3S effectors directly interfere with PRRs and their coreceptors to suppress PTI. For example, the Pseudomonas syringae pv. tomato AvrPtoB effector is a multidomain protein that acts both as E3 ligase to ubiquitinate and degrade PRRs and as an inhibitor of kinase activity of PRRs and their coreceptor BAK1 (Gimenez-Ibanez et al. 2009; Göhre et al. 2008; Shan et al. 2008; Zeng et al. 2012). PRR-associated RLCK and immunity-associated MAP kinase cascades are additional important targets for effectors that suppress PTI. Among the effectors that target RLCKs is AvrPphB from $P$. syringae pv. phaseolicola, which functions as a cysteine protease and cleaves multiple members of the BIK1 family, which are direct substrates of several PRRs (Zhang et al. 2010). A strategy used to interfere with PTI-associated MAP kinase cascades is exemplified by the action of the HopAI1 effector from $P$. syringae pv. tomato, which inactivates the MAP kinases MPK3, MPK4, and MPK6, through its phosphothreonine lyase activity (Zhang et al. 2007). Effectors can also suppress PTI by interfering with plant vesicle trafficking and protein secretion pathways that are required for secretion of immunity-associated molecules and for transport of immune receptors to the cell surface. For example, Xanthomonas euvesicatoria XopJ interferes with protein secretion to inhibit immunityassociated callose deposition at the cell wall (Bartetzko et al. 2009).

$X$. euvesicatoria is the causal agent of spot disease in pepper and tomato plants and its pathogenicity depends on the T3S system (Jones et al. 1998). To date, the pool of X. euvesicatoria T3S 
effectors includes about 35 proteins (Roden et al. 2004; Teper et al. 2016; Thieme et al. 2005). Several X. euvesicatoria effectors were reported to contribute to bacterial virulence by interfering with various PTI-associated responses (Bartetzko et al. 2009; Kim et al. 2008, 2009; Schulze et al. 2012; Singer et al. 2013; Stork et al. 2015). However, cellular targets and mechanisms of PTI inhibition are known for only a limited number of $X$. euvesicatoria effectors. The XopJ effector targets a proteasome subunit to suppress salicylic acid-mediated defense and protein secretion (Bartetzko et al. 2009; Ustün et al. 2013). The XopN effector targets the tomato 14-3-3 protein TFT1 to suppress PTI signaling (Kim et al.

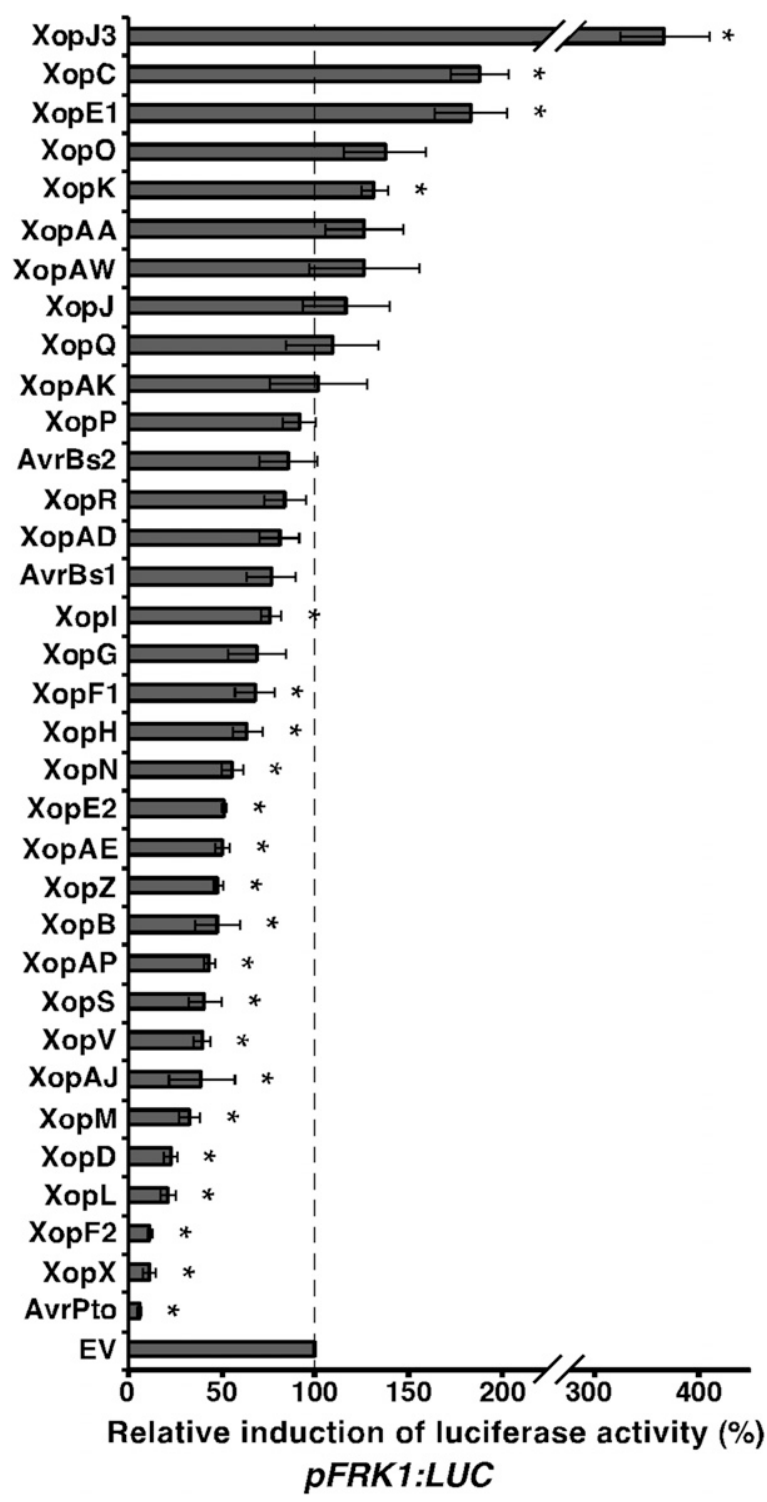

Fig. 1. Inhibition of flg22-inducible reporter gene activation by Xanthomonas euvesicatoria 85-10 type III secretion effectors. Arabidopsis mesophyll protoplasts were cotransformed with plasmids for the expression of the indicated effector or an empty vector (EV), the reporter gene $p F R K 1$ : $L U C$, and the normalizer gene $p U B Q 10: G U S$. Twelve hours after transformation, protoplasts were treated with flg22 or water. Luciferase activity was measured for $8 \mathrm{~h}$ after flg22 treatment at 2-h intervals and was normalized by $\beta$-glucuronidase activity in flg22-treated and untreated samples. For each effector, induction of the reporter gene $p F R K 1: L U C$ by flg 22 was calculated relative to that observed for the EV control $(18.7 \pm 2.6)$. Bars represent means \pm standard error of at least three independent experiments measured at the time point of maximal induction. Asterisks indicate a significant difference (Student's $t$ test, $P$ value $<0.05$ ) between the tested effector and the EV control.
2009), whereas the XopD effector encodes a SUMO protease that inhibits host transcription to suppress defense hormone signaling (Canonne et al. 2011; Kim et al. 2013).

In this study, we used Arabidopsis mesophyll protoplasts, an established pathogen-free cell system for transient gene expression analysis (Fraiture et al. 2014; Schulze et al. 2012; Shan et al. 2008; Yoo et al. 2007), to identify the entire pool of $X$. euvesicatoria 85$10 \mathrm{~T} 3 \mathrm{~S}$ effectors that interfere with flg22 defense signaling. We identified $17 \mathrm{X}$. euvesicatoria effectors that inhibited the activation of a flg22-responsive promoter acting downstream or in parallel to MAP kinase posttranslational activation. Eight of these effectors only affected flg22-induced gene expression, while nine others also interfered with abscisic acid (ABA)-dependent gene expression. Subcellular localization studies and functional characterization for a group of inhibitors of flg22 signaling support the conclusion that they contribute to bacterial virulence by targeting different steps or pathways of PTI signaling.

\section{RESULTS}

\section{A large group of $X$. euvesicatoria 85-10 T3S effectors inhibits flg22-inducible reporter gene activation in Arabidopsis protoplasts.}

Suppression of plant immunity is a prerequisite for phytopathogenic bacteria to mount a successful infection in the host (Feng and Zhou 2012). To identify X. euvesicatoria T3S effectors that suppress PTI, we utilized an Arabidopsis leaf protoplast assay, a well-established system for PAMP signaling studies (Fraiture et al. 2014) and examined the ability of 33 effectors of X. euvesicatoria 85-10 (Supplementary Table S1) to inhibit activation of gene expression induced by the bacterial peptide flg22 (Asai et al. 2002). To this aim, each effector was transiently expressed in Arabidopsis mesophyll protoplasts and was tested for its ability to inhibit activation of the flg22inducible FRK1 promoter fused to the firefly luciferase gene ( $p F R K 1: L U C)$ after application of flg22. Seven X. euvesicatoria effectors (XopX, XopL, XopD, XopS, XopB, XopN, and XopJ), which were previously reported to suppress PTI-associated responses (Bartetzko et al. 2009; Kim et al. 2008, 2009; Schulze et al. 2012; Singer et al. 2013; Stork et al. 2015), were tested again in this screen. AvrPto from P. syringae pv. tomato DC3000 was used as an additional positive control (He et al. 2006), while an empty vector was used as a negative control. Of the 33 effectors tested, 17 significantly reduced flg22-mediated activation of the pFRK1:LUC reporter gene (Fig. 1). These included six effectors (XopX, XopL, XopD, XopS, XopB, and XopN) that were already reported to interfere with PTI responses and 11 additional PTI inhibitor candidates (XopF2, XopM, XopAJ, XopV, ХopAP, XopZ, ХopAE, ХорЕ2, ХорН, ХopF1, and XopI). Four effectors (XopK, XopE1, XopC, and XopJ3) increased flg22-mediated activation of the pFRK1:LUC reporter, while 12 other effectors (XopG, AvrBs1, XopAD, XopR, AvrBs2, XopP, XорAK, XopQ, XорJ, XopAW, XopAA, and XopO) did not display any significant effect (Fig. 1).

Next, we tested whether the $17 X$. euvesicatoria effectors that reduced flg22-mediated activation of the $p F R K 1: L U C$ reporter only inhibit transcription of PTI-associated genes or also affect expression of genes involved in other cellular processes. To this aim, each effector was transiently expressed in Arabidopsis mesophyll protoplasts and was tested for its ability to inhibit activation of the ABA-inducible $R D 29 b$ promoter (Christmann et al. 2005) fused to the luciferase gene ( $p R D 29 b: L U C)$. As shown in Figure 2, eight effectors (XopZ, XopH, XopAE, XopE2, XopF1, XopAP, XopB, and XopAJ) did not inhibit activation of the $p R D 29 b: L U C$ reporter by ABA. The other nine effectors (XopF2, XopV, XopS, XopI, XopL, XopN, XopD, $\mathrm{XopM}$ and $\mathrm{XopX}$ ) significantly inhibited activation of both 
pFRK1:LUC and pRD29b:LUC reporter genes by the respective inducer (Figs. 1 and 2). These results suggest that $X$. euvesicatoria bacteria employ a large part of their T3S effector repertoire to inhibit flg22 defense signaling either selectively or by simultaneously affecting additional cellular processes.

\section{$X$. euvesicatoria T3S effectors interfere with flg22-induced} signaling downstream of MPK3 and MPK6 activation.

The Arabidopsis MAP kinases MPK3 and MPK6 have been shown to participate in early flg22 signaling and to be activated by phosphorylation (Asai et al. 2002). Thus, we tested whether $X$. euvesicatoria effectors that inhibit activation of the $p F R K 1$ : $L U C$ reporter interfere with flg22 signaling upstream or downstream of MPK3 and MPK6 activation. To this aim, hemagglutinin (HA)-tagged MPK3 or MPK6 were cotransfected into protoplasts with individual effectors and MPK3 or MPK6 activation by flg 22 treatment was monitored by Western blot analysis using p44/42 MAP kinase antibodies, which specifically recognize phosphorylated forms of MAP kinases. An empty vector, the green fluorescent protein (GFP), and the $P$. syringae pv. tomato AvrPto effector, which was previously shown to

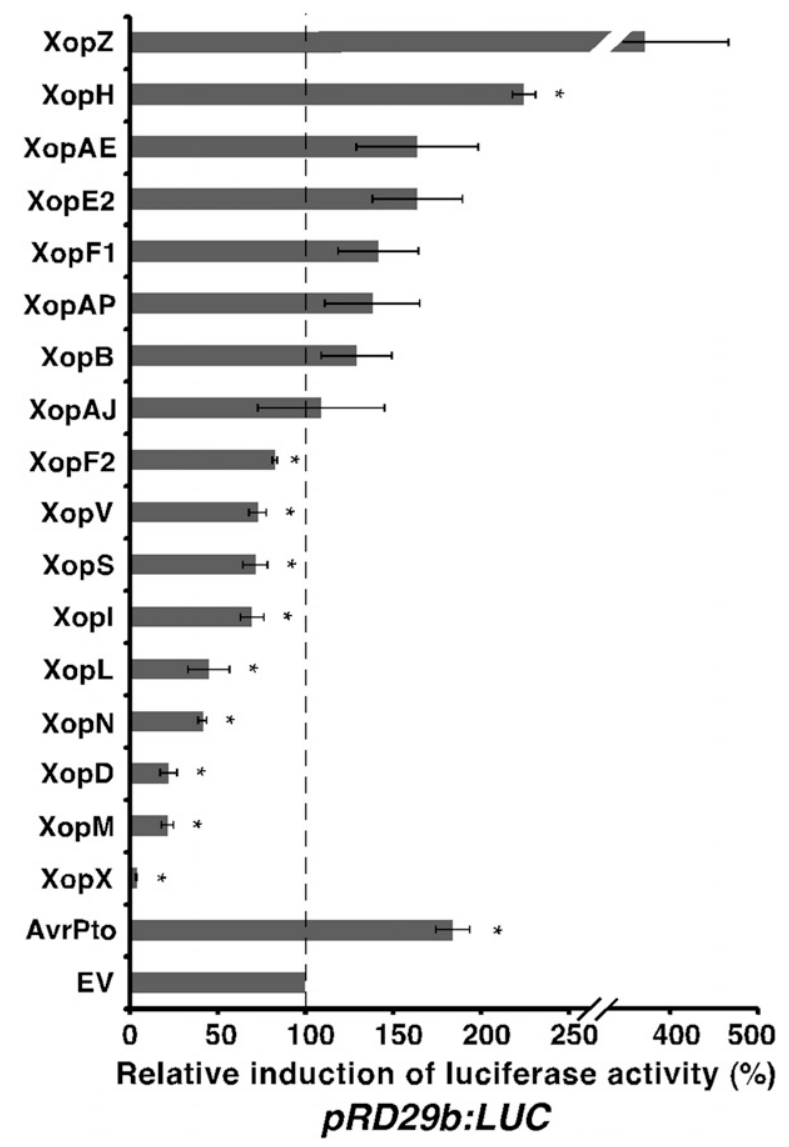

Fig. 2. Inhibition of abscisic acid (ABA)-inducible reporter gene activation by Xanthomonas euvesicatoria 85-10 type III secretion effectors. Arabidopsis mesophyll protoplasts were cotransformed with plasmids for the expression of the indicated effector or an empty vector (EV), the reporter gene $p R D 29 b: L U C$, and the normalizer gene $p U B Q 10: G U S$. Twelve hours after transformation, protoplasts were treated with $\mathrm{ABA}$ or a mock solution. Luciferase activity was measured for $8 \mathrm{~h}$ after ABA treatment at 2-h intervals and was normalized by $\beta$-glucuronidase activity in treated and untreated samples. For each effector, induction of the reporter gene $p R D 29 b$ : $L U C$ by $\mathrm{ABA}$ was calculated relative to that observed for the EV control (26.8 \pm 3.6$)$. Bars represent means \pm standard error of at least three independent experiments at the time point of maximal induction. Asterisks indicate a significant difference (Student's $t$ test, $P$ value $<0.05$ ) between the tested effector and the EV control. suppress flg22-induced activation of MPK3 and MPK6 by interfering with the activity of the FLS2 receptor (Xiang et al. 2008), were used as controls in these experiments. The XopX and XopM effectors were found to significantly inhibit general protein expression (data not shown) and were not included in this analysis. As shown in Figure 3, flg22 activated MPK3 and MPK6 phosphorylation in protoplasts transfected with an empty vector, while AvrPto suppressed this activation. Strikingly, none of the 15 X. euvesicatoria tested effectors affected MPK3 or MPK6 phosphorylation (Fig. 3, for XopF1; Supplementary Fig. S1, for the other effectors) suggesting that they inhibit flg22-induced reporter gene activation by affecting signaling mechanisms downstream or in parallel to MAP kinase activation.

Validation of the biological relevance of the Arabidopsis protoplast system for the identification of $X$. euvesicatoria effectors that suppress PTI.

Despite the fact that Arabidopsis is a X. euvesicatoria nonhost plant, we chose the Arabidopsis protoplast system to identify $X$. euvesicatoria effectors that suppress PTI because this system is much more robust than its tomato counterpart and is highly responsive to flg22 elicitation. The choice of Arabidopsis as the model plant and the PAMP flg22 as the inducer of PTI signaling
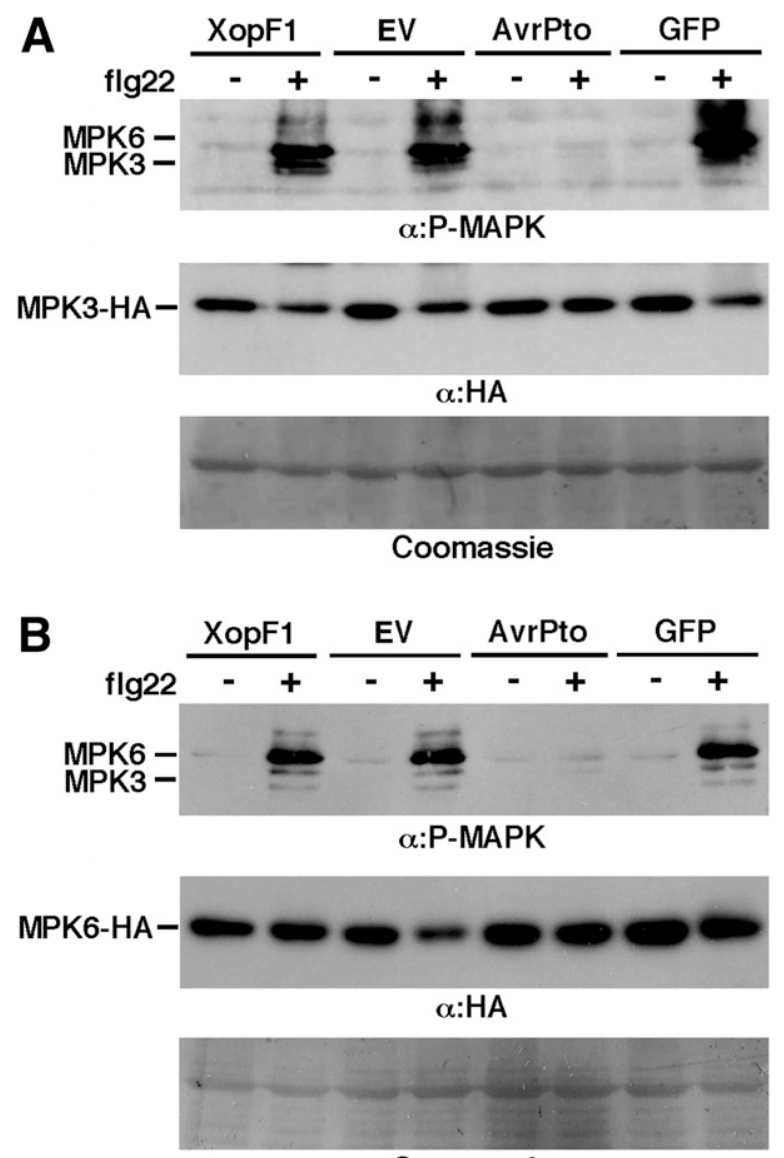

Coomassie

Fig. 3. The XopF1 effector does not affect activation of mitogen-activated protein (MAP) kinase. Arabidopsis protoplasts were cotransformed with plasmids for the expression of $\mathbf{A}$, hemagglutinin (HA)-tagged MPK3 or $\mathbf{B}$, MPK6, and XopF1, AvrPto, green fluorescent protein (GFP), or an empty vector $(\mathrm{EV})$. Twelve hours later, protoplasts were treated with flg22 (+) or mock (-) and were harvested after $5 \mathrm{~min}$. MAP kinase phosphorylation and expression of HA-tagged MAP kinases were monitored by immunoblot analysis using $\alpha:$ p44/42 MAP kinase ( $\alpha: P-M A P K)$ and $\alpha:$ HA antibodies, respectively. Coomassie staining served as loading control. The experiment was repeated three times with similar results. 
was based on the assumptions that i) $X$. euvesicatoria effectors target components of PTI signaling pathways that are conserved in Arabidopsis and tomato plants and ii) components of PTI signaling targeted by $X$. euvesicatoria effectors rely on the signal activated by recognition of flg 22 and other PAMPs as well. To confirm the correctness of the first assumption, we tested whether $X$. euvesicatoria effectors that inhibited flg22-induced gene expression in Arabidopsis protoplasts have a similar effect in tomato protoplasts. To verify the second assumption, we tested whether they inhibit gene expression induced by the bacterial PAMP elf18 (Kunze et al. 2004) in Arabidopsis protoplasts. This analysis included six genes encoding effectors that inhibited reporter gene activation induced by flg22 but not by ABA (i.e., XopAP, XopF1, XopAJ, XopAE, XopH, and XopE2), the $\mathrm{XopF} 2$ effector, which was a very strong inhibitor of flg22induced reporter gene activation but a weak inhibitor of ABAinduced gene expression, and $P$. syringae pv. tomato AvrPto as a positive control. Four of the seven $X$. euvesicatoria effectors tested (i.e., XopF2, XopE2, XopAE, and XopAJ) and AvrPto significantly inhibited flg22-induced activation of the pFRK1: $L U C$ reporter gene in tomato protoplasts (Fig. 4). In addition, three X. euvesicatoria effectors (i.e., XopF2, XopE2, and XopAJ) and AvrPto significantly reduced elf18-induced activation of the pFRK1:LUC reporter gene in Arabidopsis protoplasts (Fig. 5). These results confirm that the Arabidopsis protoplast system in combination with flg22 elicitation is reliable for the identification and further characterization of $X$. euvesicatoria effectors that suppress PTI.

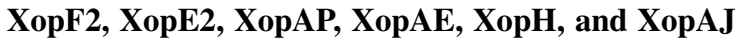 decrease callose deposition at the cell wall and enhance disease symptoms.}

To evaluate the contribution of XopF1, XopF2, XopE2, XopAP, XopAE, XopH, and XopAJ to X. euvesicatoria 85-10 virulence, we inactivated the corresponding genes by deletion

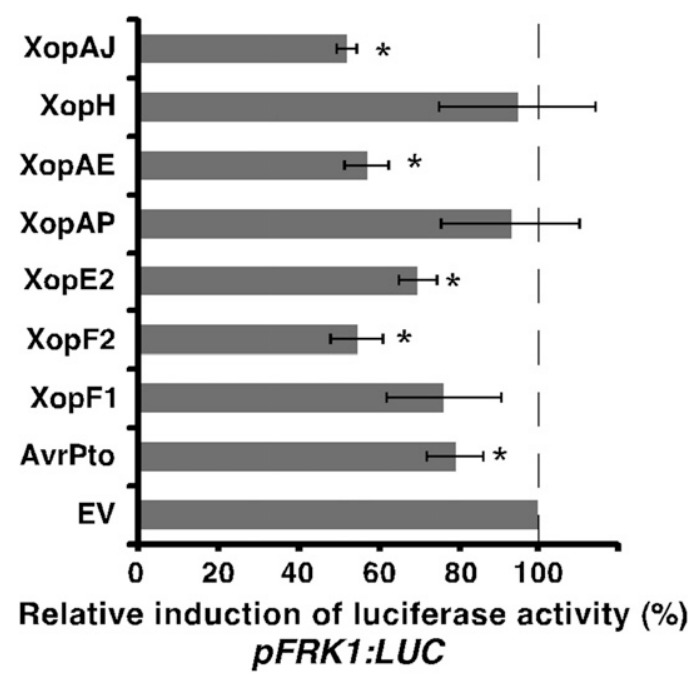

Fig. 4. Inhibition of flg22-inducible reporter gene activation by Xanthomonas euvesicatoria 85-10 type III secretion effectors in tomato. Tomato mesophyll protoplasts were cotransformed with plasmids for the expression of the indicated effector or an empty vector (EV), the reporter gene $p F R K 1: L U C$, and the normalizer gene $p U B Q 10: G U S$. Twelve hours after transformation, protoplasts were treated with flg 22 or water. Luciferase activity was measured for $6 \mathrm{~h}$ after flg22 treatment at 2-h intervals and was normalized by average $\beta$-glucuronidase activity in flg22-treated and untreated samples. For each effector, induction of the reporter gene $p F R K 1: L U C$ by flg 22 was calculated relative to that observed for the EV control $(1.8 \pm 0.1)$. Bars represent means \pm standard error of at least three independent experiments measured at the time point of maximal induction. Asterisks indicate a significant difference (Student's $t$ test, $P$ value $<0.05)$ between the tested effector and the EV control. or insertion mutagenesis. The mutant strains were used to infect susceptible pepper plants by syringe-infiltration at a titer of $1 \times 10^{5} \mathrm{CFU} / \mathrm{ml}$. Bacterial growth of the mutant strains was monitored for 8 days in the infected plants, but it did not differ from that observed for wild-type bacteria (Supplementary Fig. S2).

To circumvent the possibility that the contribution of the effectors to $X$. euvesicatoria virulence is too subtle to be detected in the original bacterial context or is masked by functional redundancy, we introduced the effectors into the attenuated $P$. syringae pv. tomato DC3000 $\triangle \mathrm{CEL}$, which lacks the AvrE and HopM1 effectors encoded by the conserved effector locus (CEL) and contains a different effector repertoire than X. euvesicatoria (Alfano et al. 2000). This strain fails to suppress callose deposition at the cell wall in Arabidopsis plants (DebRoy et al. 2004), a defense response triggered by PAMPs in infected host leaves, and it is less virulent than the wild-type $P$. syringae pv. tomato DC3000 in tomato plants (Badel et al. 2003). Individual X. euvesicatoria effectors introduced into $P$. syringae pv. tomato DC3000 $\triangle \mathrm{CEL}$ strains were tested for their effect on callose deposition in Arabidopsis and on bacterial growth and development of disease symptoms in tomato. For their expression in P. syringae pv. tomato $\triangle \mathrm{CEL}$ and translocation into host cells, the X. euvesicatoria effectors were fused downstream of the T3S translocation signal sequence of the P. syringae pv. tomato effector AvrRpt2 (located between amino acids 1 and 100; AvrRpt2 $2_{1-100}$ [Kim et al. 2009]). The $P$. syringae pv. tomato effector HopM1, which was previously shown to suppress PTI in Arabidopsis (DebRoy et al. 2004) and to contribute to P. syringae pv. tomato virulence in tomato by enhancing disease symptoms without affecting bacterial growth (Badel et al. 2003), was also introduced in the $P$. syringae pv. tomato DC3000 $\triangle \mathrm{CEL}$ strain and served as a positive control in these experiments. To test the effect of

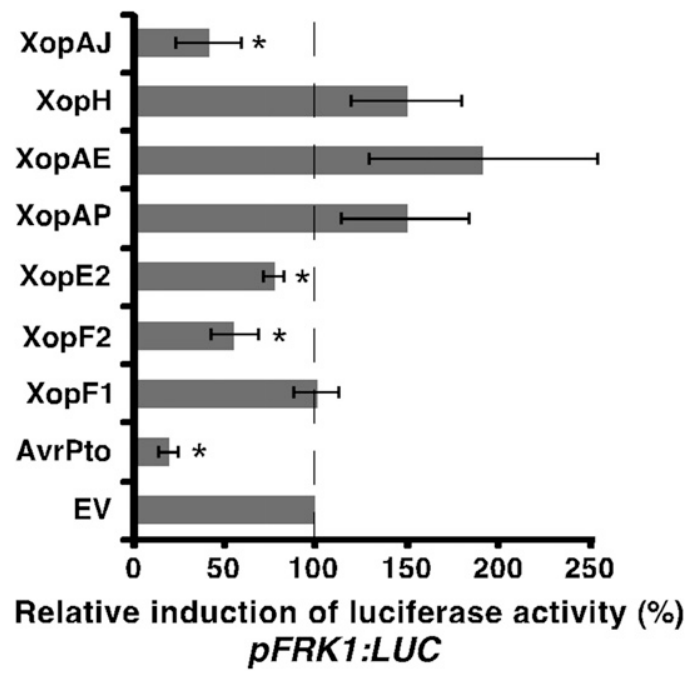

Fig. 5. Inhibition of elf18-inducible reporter gene activation by Xanthomonas euvesicatoria 85-10 type III secretion effectors. Arabidopsis mesophyll protoplasts were cotransformed with plasmids for the expression of the indicated effector or an empty vector (EV), the reporter gene $p F R K 1$ : $L U C$, and the normalizer gene $p U B Q 10: G U S$. Twelve hours after transformation, protoplasts were treated with elf 18 or water. Luciferase activity was measured for $8 \mathrm{~h}$ after elf18 treatment at 2-h intervals and was normalized by $\beta$-glucuronidase activity in elf18-treated and untreated samples. For each effector, induction of the reporter gene $p F R K 1: L U C$ by elf 18 was calculated relative to that observed for the EV control $(8.8 \pm 2.5)$. Bars represent means \pm standard error of at least three independent experiments measured at the time point of maximal induction. Asterisks indicate a significant difference (Student's $t$ test, $P$ value $<0.05$ ) between the tested effector and the EV control. 
effectors on callose deposition, Arabidopsis Col-0 leaves were hand-infiltrated with a suspension $\left(2 \times 10^{8} \mathrm{CFU} / \mathrm{ml}\right)$ of $P$. syringae pv. tomato $\Delta$ CEL expressing HopM1, AvrRpt $2_{1-100}$, or AvrRpt $2_{1-100}$-effector fusions and were stained for callose after $16 \mathrm{~h}$. As expected, Arabidopsis leaves infected with $P$. syringae pv. tomato $\triangle \mathrm{CEL}$ expressing the HopM1 effector had fewer callose deposits as compared with leaves infiltrated with $P$. syringae pv. tomato $\triangle \mathrm{CEL}$ expressing AvrRpt $2_{1-100}$ (Fig. 6). Similarly, fewer callose deposits accumulated in leaves infected with $P$. syringae pv. tomato $\triangle \mathrm{CEL}$ expressing all the tested AvrRpt21-100-effector fusions except for AvrRpt2 1-100-XopF1 (Fig. 6).

Next, to test the contribution of the $X$. euvesicatoria effectors to $P$. syringae pv. tomato $\triangle \mathrm{CEL}$ virulence, tomato plants were infiltrated with a mock solution or with a suspension $\left(1 \times 10^{5}\right.$ $\mathrm{CFU} / \mathrm{ml}$ ) of $P$. syringae pv. tomato $\triangle \mathrm{CEL}$ bacteria expressing HopM1, AvrRpt2 $1-100$, or an AvrRpt2 1-100-effector fusion and development of disease symptoms and bacterial growth were monitored for 3 days postinoculation (dpi). P. syringae pv. tomato $\triangle$ CEL expressing HopM1, AvrRpt2 1 100 fused to XopF2, XopE2, XopAP, XopAE, XopH, and XopAJ, but not XopF1 caused more severe disease symptoms than $P$. syringae pv. tomato $\triangle \mathrm{CEL}$ expressing AvrRpt $2_{1-100}$ (Fig. 7A and B). Despite the effect of the tested effectors on disease symptoms, bacterial populations were similar for all the strains at $3 \mathrm{dpi}$ (Supplementary Fig. S3). As assessed by Western blot analysis (Supplementary Fig. S4), HopM1 and the XopF1, XopF2, XopE2, XopAE, and XopAJ effectors fused to AvrRpt $2_{1-100}$ were detected and expressed at different levels in $P$. syringae pv. tomato $\triangle \mathrm{CEL}$ strains, while expression of the XopAP and $\mathrm{XopH}$ effectors fused to AvrRpt $2_{1-100}$ was below detection levels. These results confirmed that effectors that inhibited flg22- but not ABA-inducible reporter gene activation in protoplasts act as PTI inhibitors in planta and contribute to development of disease symptoms.

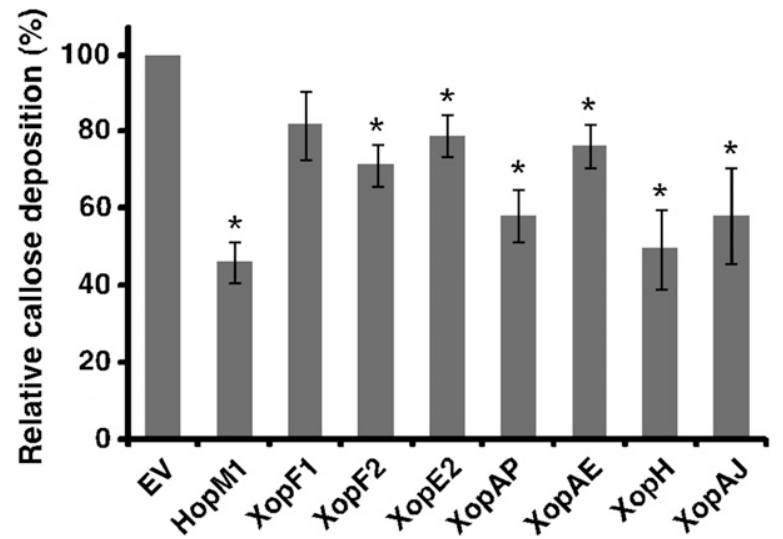

Fig. 6. Inhibition of flg22-induced callose deposition by Xanthomonas euvesicatoria type III secretion effectors in Arabidopsis plants. Arabidopsis leaves were inoculated with Pseudomonas syringae pv. tomato DC3000 $\triangle \mathrm{CEL}$ strains $\left(1 \times 10^{8} \mathrm{CFU} / \mathrm{ml}\right)$ expressing the indicated $X$. euvesicatoria effectors or an empty vector (EV). Sixteen hours after inoculation, leaves were cleared and stained with aniline blue, and callose deposits were visualized in four fields $\left(0.6 \mathrm{~mm}^{2}\right)$ by fluorescence microscopy and were counted. In each experiment, the number of callose deposits counted in mock-inoculated leaves was used as background and was subtracted from the mean of each treatment. Callose deposition is reported as the percentage of that observed in leaves inoculated with the $P$. syringae pv. tomato DC3000 $\triangle$ CEL strain carrying an EV (172.4 \pm 13.2 deposits/ $0.6 \mathrm{~mm}^{2}$ ). Data are the means \pm standard error of at least three independent biological replicates. Asterisks indicate statistically significant differences (Student's $t$ test, $P$ value $<0.05$ ) between the tested effectors and the EV control.

$X$. euvesicatoria effectors that inhibited flg22 defense signaling localize to different subcellular compartments.

The subcellular localization of effectors in the host may provide important clues about the effector molecular functions. To investigate whether $X$. euvesicatoria effectors that suppress

A
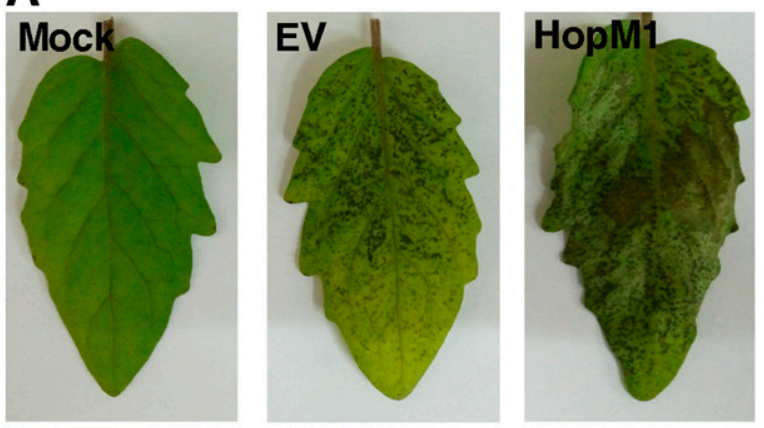

B
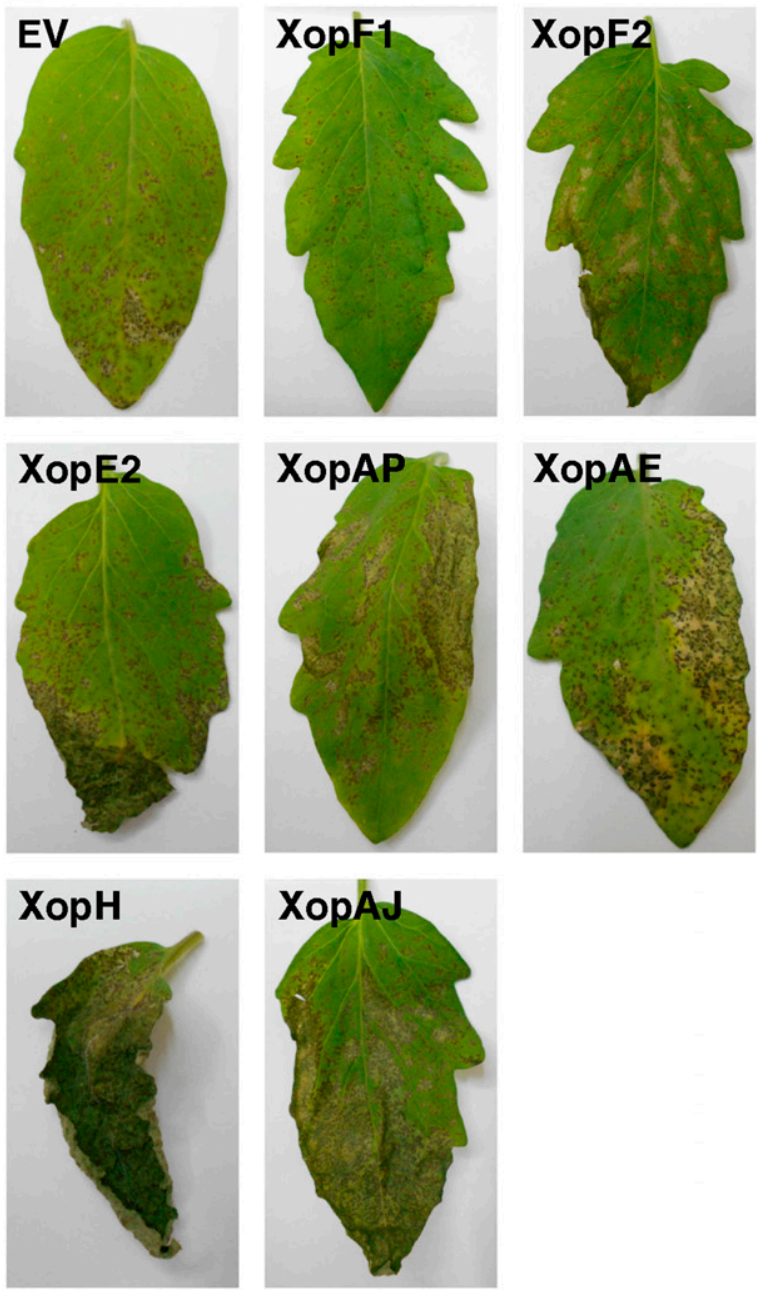

Fig. 7. The XopF2, XopE2, XopAP, XopAE, XopH and XopAJ effectors contribute to disease symptoms caused by Pseudomonas syringae pv. tomato DC3000 $\triangle$ CEL bacteria in tomato plants. Tomato leaves were mock inoculated (Mock) or vacuum-infiltrated with suspensions $\left(1 \times 10^{5}\right.$ $\mathrm{CFU} / \mathrm{ml}$ ) of $P$. syringae pv. tomato DC3000 $\Delta \mathrm{CEL}$ strains expressing an empty vector (EV), A, the P. syringae pv. tomato effector HopM1, or B, the indicated Xanthomonas euvesicatoria effectors. Inoculated leaves were monitored for the appearance of disease symptoms and photographed at 3 days postinoculation. The experiment was repeated three times with similar results. 
PTI target similar or different molecular mechanisms, we determined subcellular localization for a group of $X$. euvesicatoria effectors that were identified in this study as inhibitors of flg22 defense signaling. In this group were the XopF1, XopAJ,
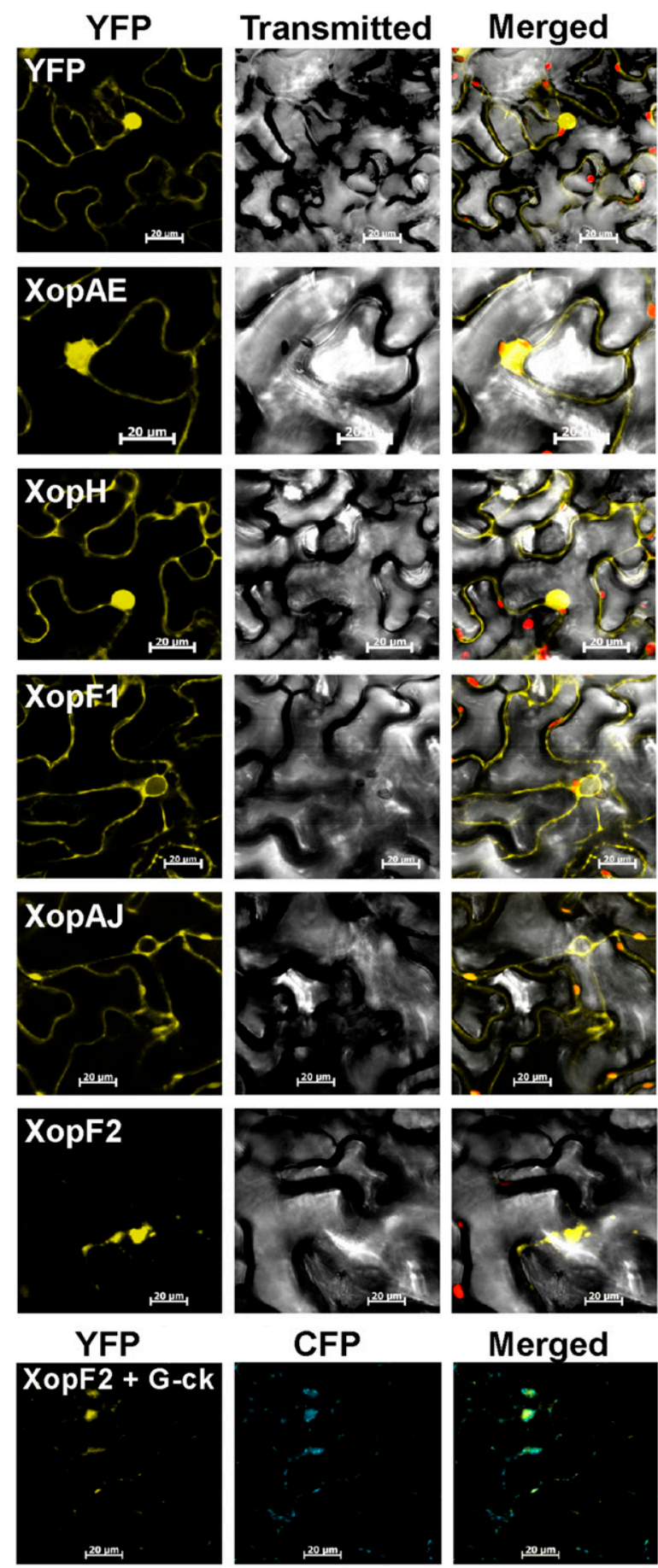

Fig. 8. Subcellular localization of the XopAE, XopH, XopF1, XopAJ, and XopF2 effectors. Agrobacterium strains expressing the indicated effectors fused to the N-terminus of yellow fluorescent protein (YFP) were infiltrated in leaves of Nicotiana benthamiana plants. In the upper panel, the fluorescence (YFP), light transmitted, and merged images are shown for each effector; in the lower panel, fluorescence distribution of XopF2-YFP and G-ck, a cyan fluorescent protein (CFP) marker of the Golgi apparatus. YFP, CFP, and merged fluorescence images are shown. Distribution of YFP fluorescence was determined at $24 \mathrm{~h}$ (for YFP, XopAE, XopH, and XopF1, whose expression is driven by the $35 \mathrm{~S}$ Cauliflower mosaic virus promoter) or $36 \mathrm{~h}$ (XopAJ, XopF2, and G-ck, whose expression is driven by the pER8 system) postinoculation, by confocal microscopy. Scale bars in each panel represent $20 \mu \mathrm{m}$.
XopAE, and XopH effectors that inhibited reporter gene activation induced by flg22 but not by $\mathrm{ABA}$ and the XopF2 effector, which is a very strong inhibitor of flg22-induced reporter gene activation but a weak inhibitor of ABA-induced gene expression. The yellow fluorescent protein (YFP) was fused to the C-terminus of the effectors and expression of the fusion proteins was driven either by the Cauliflower mosaic virus (CaMV) 35S promoter (for XopF1, XopAE, and XopH) or by an estradiol-inducible expression system (for XopF2 and XopAJ). The obtained fusion proteins and a YFP control were transiently expressed via Agrobacterium tumefaciens in leaves of $N$. benthamiana plants, and their subcellular localization in lower epidermal cells was determined by confocal laser scanning microscopy. YFP, XopAE-YFP, and XopH-YFP showed a similar subcellular localization, and their fluorescence was detectable in the cytoplasm and in the nucleus (Fig. 8, upper panel). Fluorescence of XopF1-YFP and XopAJ-YFP was detected in the cytoplasm but not in the nucleus (Fig. 8, upper panel). To confirm cytoplasmic localization for XopAE-YFP, XopH-YFP, XopF1-YFP, and XopAJ-YFP, the effector fusions were coexpressed with the cyan fluorescent protein (CFP), which localizes to the cytoplasm and the nucleus (Kruse et al. 2010). As expected, XopAE-YFP and XopH-YFP showed complete colocalization with CFP, while XopF1-YFP and XopAJ-YFP colocalized with CFP in the cytoplasm but not in the nucleus (Supplementary Fig. S5). XopF2-YFP fluorescence was confined to small structures in the cytoplasm and to a few larger structures, mainly surrounding the nucleus. Because this pattern is consistent with localization to the Golgi apparatus, we transiently coexpressed XopF2-YFP with G-ck, a CFP marker for the Golgi apparatus (Nelson et al. 2007). Colocalization of the two proteins confirmed that the XopF2 effector localizes to the Golgi apparatus (Fig. 8, lower panel). These results demonstrate that $X$. euvesicatoria effectors sharing the ability to inhibit flg22 defense signaling localize to various compartments of the host cells, implying that individual effectors target different steps or pathways of PTI signaling.

\section{DISCUSSION}

In this study, we employed an Arabidopsis protoplast transient expression system for identifying the repertoire of $X$. euvesicatoria 85-10 effectors that interfere with PTI. The PAMP used in our investigation is flg22, a 22-amino acid peptide corresponding to the highly conserved amino terminus of flagellin that is sufficient to trigger immune responses in several plant species (Felix et al. 1999). The cell-based protoplast system provides a fast and reliable experimental platform allowing testing of each individual effector independently of the original bacterial context that may mask subtle or redundant contribution of the effector to PTI suppression. Similar screens were performed in protoplasts to identify $P$. syringae pv. tomato and Phytophthora infestans effectors that interfere with flg22 defense signaling (He et al. 2006; Zheng et al. 2014) and to provide evidence that the $X$. euvesicatoria effectors XopB, XopS, and XopL inhibit flg22- and elf18-induced activation of a PAMP-inducible promoter (Schulze et al. 2012; Singer et al. 2013).

Of the $33 X$. euvesicatoria 85-10 effectors tested in our analysis, 17 (approximately 50\%) inhibited and four enhanced the induction of a flg22-inducible reporter gene construct. It is tempting to speculate that effectors that enhanced reporter induction are recognized in Arabidopsis cells and prime immune signaling. Effectors that inhibited reporter induction represent a large proportion of the known effector pool of the $X$. euvesicatoria 85-10 bacterial strain, indicating that suppression of PTI is a primary role of $X$. euvesicatoria effectors. This finding together with the observation that several X. euvesicatoria effectors (i.e., AvrBsT, 
XopE1, XopM, XopQ, AvrBs1, AvrXv4) were previously shown to inhibit cell death associated with effector-triggered immunity (ETI) (Szczesny et al. 2010; Teper et al. 2014, 2016) support the notion that suppression of different branches of plant immunity at different levels is a fundamental requirement for $X$. euvesicatoria bacteria to colonize their host. In line with this observation, multiple effectors of the same pathogen were previously implicated in suppression of plant immunity. For example, a large group of $P$. syringae pv. tomato effectors was reported to suppress PTI and ETI responses (Guo et al. 2009; He et al. 2006; Jamir et al. 2004). Similarly, eight of 33 tested Phytophthora infestans effectors were shown to inhibit flg22-induced gene activation (Zheng et al. 2014).

Six of the $17 \mathrm{X}$. euvesicatoria effectors that were found to inhibit flg22 reporter gene activation (i.e., XopL, XopX, XopB, XopS, XopN, and XopD) were previously shown to interfere with various PTI responses. XopN was reported to reduce PAMP-induced gene expression and callose deposition in tomato plants (Kim et al. 2009), whereas XopD was shown to interfere with activation of defense gene expression induced by the plant hormones salicylic acid and ethylene in tomato (Kim et al. 2008). In addition, the XopB, XopS, and XopL effectors were found to inhibit PAMP-induced activation of the NHL1O promoter in Arabidopsis protoplasts (Schulze et al. 2012; Singer et al. 2013). Finally, XopX was recently shown to suppress flg22-induced production of reactive oxygen species but to promote transcriptional activation of PTI-associated genes (Stork et al. 2015). Our findings provide further evidence for the role of these effectors in modulation of plant immunity. Moreover, the detection of a phenotype for effectors that were already linked to suppression of PTI responses in the host plant validated the use of the Arabidopsis protoplast transient expression system for the functional analysis of the entire pool of $X$. euvesicatoria effectors. The XopJ effector was previously shown to interfere with cell wall-associated defense responses (Bartetzko et al. 2009) but did not inhibit flg22-induced reporter gene activation in our analysis. It is possible that XopJ affects a pathway of PTI signaling that does not lead to FRK1 transcriptional activation or that it targets molecules involved in the execution rather than in signaling of immune responses.

Eleven $X$. euvesicatoria effectors that inibited flg22-inducible reporter gene activation (i.e., XopF2, XopM, XopAJ, XopV, XopAP, XopZ, XopAE, XopE2, XopH, XopF1, and XopI) have not been previously linked to suppression of plant immunity and their molecular mode of action is still largely unknown. Of the 17 effectors that inhibited flg22-inducible reporter gene activation, eight effectors inhibited only PTI, while the other nine also interfered with ABA-dependent activation of a reporter gene driven by an ABA-inducible promoter. It is interesting to note that the effectors identified in this study as inhibitors of flg22 reporter gene activation did not affect flg22-induced MAP kinase phosphorylation, indicating that none of them interferred with the function of the FLS2 receptor or components of the FLS2 receptor complex. This is different from what has been observed in similar screens for P. syringae pv. tomato and Phytophthora infestans effectors (He et al. 2006; Zheng et al. 2014). The P. syringae pv. tomato effectors AvrPto and AvrPtoB were first shown to suppress flg22 defense signaling upstream to the activation of MAP kinase cascades (He et al. 2006), and follow-up studies demonstrated that they interfere with the function of PRR receptors and coreceptors (Gimenez-Ibanez et al. 2009; Göhre et al. 2008; Shan et al. 2008; Xiang et al. 2008, 2011; Zeng et al. 2012). Similarly, three Phytophthora effectors (SFI5, SFI6, and SFI7) were found to inhibit flg22-induced phosphorylation of MAP kinases (Zheng et al. 2014). However, it is still possible that certain $X$. euvesicatoria effectors affect the functions of PRRs other than FLS2 or that of early signaling components that do not participate in flg22 defense signaling. In addition, it is possible that certain $X$. euvesicatoria effectors interfere with the function of early signaling components that are specific to the host plants pepper and tomato and are absent or not sufficiently conserved in the nonhost Arabidopsis. In support of this hypothesis, the Phytophthora effectors SFI5, SFI6, and SFI7 attenuated flg22induced phosphorylation of MAP kinases in tomato but not in Arabidopsis protoplasts (Zheng et al. 2014).

Mutations in individual genes encoding effectors that inhibited flg22-induced PTI but not ABA-inducible gene expression (i.e., XopE2, XopF1, XopF2, XopH, XopAP, ХopAE, and XopAJ) did not cause reduction of $X$. euvesicatoria virulence in pepper plants. This is not unexpected and is probably due to the functional redundancy often observed within the pool of T3S effectors of a given bacterial strain. However, when performing gain-of-function experiments through heterologous expression in the attenuated $P$. syringae pv. tomato $\triangle \mathrm{CEL}$ strain, the expression of all the effectors tested, except for XopF1, inhibited callose deposition at the plant cell wall in Arabidopsis and enhanced development of disease symptoms in tomato. Despite the redundancy in their contribution to PTI suppression, the various effectors that inhibited flg22-induced PTI appear to act at different levels and by different mechanisms, as they were found in this and previous studies to localize to various cellular compartments (Bartetzko et al. 2009; Schulze et al. 2012; Thieme et al. 2007). Noteworthy is the localization of XopF2 to the Golgi apparatus. A similar localization was observed for the XopJ and XopB effectors that interfere with PTI-associated responses and inhibit vesicle trafficking (Bartetzko et al. 2009; Schulze et al. 2012). It remains to be established whether these effectors share redundant functions by affecting similar cellular processes.

In conclusion, this study identified 11 X. euvesicatoria $85-10$ effectors as PTI inhibitors, providing molecular and physiological phenotypes for further investigation of their molecular function and interference with plant immunity.

\section{MATERIALS AND METHODS}

\section{Bacterial strains and plant material.}

Bacteria used were Escherichia coli DH12S, Agrobacterium tumefaciens GV2260, X. euvesicatoria 85-10 (Thieme et al. 2005), and Pseudomonas syringae pv. tomato DC3000 $\Delta \mathrm{CEL}$ (Alfano et al. 2000). Bacteria were grown in Luria Bertani broth supplemented with the appropriate antibiotics at the following temperatures: E. coli at $37^{\circ} \mathrm{C}$, A. tumefaciens, X. euvesicatoria, and $P$. syringae pv. tomato at $28^{\circ} \mathrm{C}$. Antibiotics were used at the following concentrations $(\mu \mathrm{g} / \mathrm{ml})$ : ampicillin, 100; kanamycin, 50; rifampicin, 100; spectinomycin, 50; streptomycin, 100; nitrofurantion, 50; and gentamicin, 10. Bacterial growth assays were performed as described (Teper et al. 2014). Bacterial strains were injected with a needless syringe into pepper leaves or vacuum-infiltrated into tomato leaves at a concentration of $1 \times 10^{5} \mathrm{CFU} / \mathrm{ml}$ in $10 \mathrm{mM} \mathrm{MgCl}{ }_{2}$, and bacterial growth was monitored at different times postinoculation.

Plant cultivars used were Arabidopsis thaliana ecotype Col-0, pepper (Capsicum annuum) 'ECW-30R' (Minsavage et al. 1990), tomato (Solanum lycopersicum) 'Hawaii 7981' (Scott et al. 1995), and Nicotiana benthamiana (Goodin et al. 2008). Arabidopsis plants were grown in a phytochamber in short day conditions $(8 \mathrm{~h}$ light and $16 \mathrm{~h}$ dark) at $22^{\circ} \mathrm{C}$ with 40 to $60 \%$ humidity and approximately $120 \mu \mathrm{E} \mathrm{m}^{-2} \mathrm{~s}^{-1}$ light intensity. Tomato, pepper, and $N$. benthamiana plants were grown in a greenhouse in long-day conditions (16 h light and $8 \mathrm{~h}$ dark) at $25^{\circ} \mathrm{C}$.

\section{Plasmid constructions.}

For expression in protoplasts, the $X$. euvesicatoria effector genes were polymerase chain reaction (PCR)-amplified, using Ex-Taq DNA polymerase (Takara Bio, Madison, WI, U.S.A.) 
from $X$. euvesicatoria 85-10 genomic DNA. Sequences of oligonucleotides used in this study for PCR amplification are available upon request. Amplified effectors were inserted in frame to a c-myc tag into the multiple cloning site of the pGML10 vector (RIKEN, Tokyo). C-myc tagged effector genes were then excised from the pGML10 vector using restriction enzymes or were PCRamplified and were inserted into the pTEX vector (Frederick et al. 1998) under the control of the CaMV 35S promoter.

For subcellular localization studies, an optimized version of the YFP (Griesbeck et al. 2001) and CFP (Nelson et al. 2007) were PCR-amplified and were cloned into the pGML10 vector either alone or in frame to the $3^{\prime}$ end of the xopH, xopAJ, xopF1, $x o p F 2, x о p A P$, and $x o p A E$ genes. The effector-YFP gene fusions and CFP were then excised from the pGML10 vector using restriction enzymes or were PCR-amplified and were inserted into the pBTEX binary vector under the control of the CaMV 35S promoter (Frederick et al. 1998) or into the $\mathrm{pER} 8$ binary vector for estradiol-inducible expression (Zuo et al. 2000).

For generation of $X$. euvesicatoria 85-10 deletion mutant strains of the $x о p H, x o p F 1, x o p F 2, x о p A P$, and $x о p E 2$ genes, a 1-kb fragment upstream and a 1-kb fragment downstream of each effector gene were PCR-amplified from the $X$. euvesicatoria $85-10$ genome and were cloned into the pGML10 vector. The gentamicin resistance cassette was then PCR-amplified from the pBBR1MCS-5 plasmid (Kovach et al. 1995) and was cloned into pGML10 between the two fragments. The obtained cassettes were excised from the pGML10 vector and were cloned into the pVIK165 suicide vector (Kalogeraki and Winans 1997). For generation of $X$. euvesicatoria 85-10 insertion mutant strains of the xopAJ and xopAE genes, a 250-bp fragment of the effector gene (nucleic acids 50 to 300 ) was PCRamplified from the X. euvesicatoria 85-10 genome and was cloned into the pVIK165 suicide vector. Suicide vectors were mobilized from $E$. coli into $X$. euvesicatoria $85-10$ by triparental mating (Rott et al. 1996). Mutants were selected with the appropriate antibiotics and were verified by PCR.

For expression of $X$. euvesicatoria effectors in the $P$. syringae pv. tomato DC3000 $\triangle$ CEL strain, a HA epitope tag was cloned into the pVSP61 (avrRpt2 1 100) vector (Kim et al. 2009) creating pVSP61(avrRpt $\left.2_{1-100}: H A\right)$. Effector genes were then PCRamplified and were cloned into pVSP61(avrRpt $\left.2_{1-100}: H A\right)$ in frame to avrRpt $2_{1-100}$ and the HA tag, resulting in pVSP61

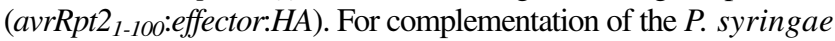
pv. tomato DC3000 $\triangle \mathrm{CEL}$ strain, the hopM1 gene was PCRamplified and was cloned into pVSP61(avrRpt2 $\left.{ }_{1-100}: H A\right)$, replacing the avrRpt $2_{1-100}$ fragment and in frame to the HA tag, resulting in pVSP61(hopM1:HA). The obtained vectors were mobilized from $E$. coli into $P$. syringae pv. tomato DC3000 $\triangle \mathrm{CEL}$ by triparental mating.

\section{Protoplast preparation and transfection.}

The preparation of Arabidopsis mesophyll protoplasts was performed as described by Fraiture et al. (2014), with minor changes. Briefly, leaf stripes were dipped into $1.5 \%$ cellulose 'Onozuka' R10 and 0.4\% macerozyme R10 solution (Yakult Pharmaceutical Industry, Tokyo), were vacuum-infiltrated for $30 \mathrm{~min}$, and were digested for $3 \mathrm{~h}$ at $20^{\circ} \mathrm{C}$ in the dark. Next, protoplasts were filtered through a 45 to $100-\mu \mathrm{m}$ nylon mesh, were washed twice with W5 buffer, and were suspended in MMG buffer (4 mM MES, pH 5.7, 0.4 M mannitol, $15 \mathrm{mM} \mathrm{MgCl}_{2}$ ) to a density of $2 \times 10^{5}$ cells $/ \mathrm{ml}$. Polyethylene glycol-mediated transfection was performed at a total DNA concentration of $100 \mu \mathrm{g}$ of plasmid DNA per milliliter of protoplast suspension. Protoplast samples were then incubated in $\mathrm{W} 1$ buffer at $20^{\circ} \mathrm{C}$ in the dark for 12 to $14 \mathrm{~h}$, allowing plasmid gene expression.

Tomato mesophyll protoplasts were prepared as described by Nguyen et al. (2010), with minor changes. The lower epidermis of fully expanded leaflets was gently rubbed with Carborundum and was rinsed with sterile water and leaf strips were floated on enzyme solution containing 2\% cellulose 'Onozuka' R10, 0.4\% macerozyme R10 (Yakult Pharmaceutical Industry) and $0.4 \mathrm{M}$ sucrose in $\mathrm{K} 3$ medium. After 30 min of vacuum-infiltration and $3 \mathrm{~h}$ of incubation at $30^{\circ} \mathrm{C}$ in the dark, the enzyme-protoplast mixture was filtered through a 45 to $100-\mu$ m nylon mesh. Viable protoplasts were collected by sucrose gradient centrifugation and were washed in W5 buffer. After recovery on ice for $2 \mathrm{~h}$, protoplasts were harvested by centrifugation and were suspended to a density of $6 \times 10^{5}$ cells $/ \mathrm{ml}$ in MMG buffer, prior to polyethylene glycol-mediated transfection that was performed at a total DNA concentration of $100 \mu \mathrm{g}$ of plasmid DNA per milliliter of protoplast suspension. Protoplast samples were then incubated in $\mathrm{W} 1$ buffer at $25^{\circ} \mathrm{C}$ in the dark for 12 to $14 \mathrm{~h}$, allowing plasmid gene expression.

\section{Luciferase and $\beta$-glucuronidase (GUS) assays.}

Luciferase and GUS assays were performed as described by Zheng et al. (2014), with minor changes. Protoplasts were cotransfected with the reporters $p F R K 1: L U C$ (inducible by flg22 and elf18 [Asai et al. 2002; Albert et al. 2010]) or pRD29b:LUC (inducible by ABA [Christmann et al. 2005]), $p U B Q 10: G U S$ (for normalization of transformation efficiency, viability, and protein expression) and a pTEX vector containing a 35S:effector gene construct, an empty pTEX vector (negative control), or a p2FGW7 vector containing a 35S: avrPto gene construct (positive control) (Zheng et al. 2014). For the luciferase assay, luciferin was added to a $600-\mu 1$ transfected protoplast solution to a final concentration of $200 \mu \mathrm{M}$. Protoplasts $(100 \mu \mathrm{l})$ were transferred to six wells in an opaque 96well plate. For each sample, a PAMP (flg22 or elf18) or ABA was added to three wells to a final concentration of $500 \mathrm{nM}$ or $50 \mu \mathrm{M}$, respectively. The remaining three wells were mocktreated with $1 \mu \mathrm{l}$ of water or ethanol as control of the PAMP and ABA treatment, respectively. The luminescence reflecting the luciferase activity was measured every $2 \mathrm{~h}$ for $8 \mathrm{~h}$, using a Turner Biosystems Veritas luminometer (Promega, Madison, WI, U.S.A.). For the GUS assay, $50 \mu 1$ of transfected protoplast solution for each sample was treated with $500 \mathrm{nM}$ PAMP or $50 \mu \mathrm{M}$ ABA, and $50 \mu \mathrm{l}$ were mock-treated. Protoplast pellets were collected 4 (tomato) or $6 \mathrm{~h}$ (Arabidopsis) after elicitation. The cells were lysed in $100 \mu \mathrm{l}$ of cell culture lysis reagent solution (Promega). For each sample, three technical replicates of $10 \mu \mathrm{l}$ were incubated with $90 \mu \mathrm{l}$ of MUG substrate $(1 \mathrm{mM}$ 4-methyl-umbelliferyl- $\beta$-D-glucuronide, $100 \mathrm{mM}$ Tris- $\mathrm{HCl}, \mathrm{pH}$ $8.0,2 \mathrm{mM} \mathrm{MgCl}_{2}$ ) for $30 \mathrm{~min}$ at $37^{\circ} \mathrm{C}$. The reaction was stopped with $90 \mu \mathrm{l}$ of $0.2 \mathrm{M} \mathrm{Na}_{2} \mathrm{CO}_{3}$. The fluorescence was monitored in an opaque plate, using a Turner Designs 20e luminometer 96-well plate reader with $\lambda_{\mathrm{ex}}=360 \mathrm{~nm}$ and $\lambda_{\mathrm{em}}=$ $460 \mathrm{~nm}$. For Arabidopsis protoplast assays, induction rate was calculated as (elicitor luciferase/elicitor GUS)/(mock luciferase/ mock GUS) in each biological repeat. For tomato protoplast assays, induction rate was calculated as (elicitor luciferase/mock luciferase) in each biological repeat and was normalized by average GUS ratio (elicitor GUS/mock GUS).

\section{Detection of phosphorylated MAP kinase.}

Arabidopsis mesophyll protoplasts were cotransformed with pUC19 containing a 35S:MPK3 or 35S:MPK6 gene construct along with a pTEX vector containing a 35S:effector gene construct, pTEX empty vector, a p2FGW7 vector containing a $35 S: G F P$ gene construct (Fraiture et al. 2014), or a p2GW7 vector containing a 35S:avrPto gene construct (Fraiture et al. 2014). For each effector tested, $1 \mathrm{ml}$ of protoplasts was distributed into two tubes and were treated with $500 \mathrm{nM}$ flg22 or water for $5 \mathrm{~min}$. Pellets of protoplasts were collected and were 
denatured in protein-loading buffer. Proteins were fractionated by sodium dodecyl sulfate-polyacrylamide gel electrophoresis and were blotted onto a polyvinylidene diflouride membrane. Phosphorylation of MAPKs was monitored by immunoblot analysis using an antiphospho-p42/44 MAP kinase antibody (Cell Signaling Technology, Danvers, MA, U.S.A.).

\section{Agrobacterium-mediated transient expression and localization assay.}

pBTEX and pER8 plasmids carrying genes encoding either YFP or an $X$. euvesicatoria effector-YFP fusion were transformed into A. tumefaciens GV2260 by electroporation. Agrobacterium strains were grown overnight at $28^{\circ} \mathrm{C}$, were diluted to an optical density at $600 \mathrm{~nm}=0.1$ in $\mathrm{MgCl}_{2}$ and were syringe-infiltrated into leaves of six-week-old $N$. benthamiana plants. Leaf areas infiltrated with A. tumefaciens carrying the pER8 plasmids were treated with estradiol at $24 \mathrm{~h}$ after infiltration. Protein localization was visualized by a confocal laser scanning microscope (LSM 510 META; Zeiss, Heidelberg, Germany). Images were processed with AxioVision software (Zeiss). The Golgi marker G-ck labeled with CFP was used for colocalization experiments (Nelson et al. 2007). YFP and chlorophyll were excited with an Argon laser at $488 \mathrm{~nm}$, while CFP was excited with a Diode laser at $405 \mathrm{~nm}$. Emission was detected with a spectral detector set between 420 and $490 \mathrm{~nm}$ for CFP, between 505 and $550 \mathrm{~nm}$ for YFP, and between 635 and $750 \mathrm{~nm}$ for chlorophyll.

\section{Callose deposition assay.}

$P$. syringae pv. tomato DC3000 $\triangle \mathrm{CEL}$ strains carrying the pVSP61 vector for expression of effector genes were syringeinfiltrated at a concentration of $1 \times 10^{8} \mathrm{CFU} / \mathrm{ml}$ into Arabidopsis leaves. Callose deposition assays were performed as described (Kim and Mackey 2008). At 16 h after infiltration, leaves were cleared of chlorophyll, callose deposits were stained with an aniline blue solution $(0.01 \%)$ and were visualized by a fluorescence microscope with Axio and AxioVision softwares (Zeiss), with excitation at $365 \mathrm{~nm}$ and emission at $445 \mathrm{~nm}$. For each treatment, a leaf from each of three plants was inoculated and four fields $\left(0.6 \mathrm{~mm}^{2}\right)$ were photographed for each leaf. The number of callose deposits (9.75 to $188.5 \mu \mathrm{m}$ in diameter) in the photographed fields was automatically counted, using the Icy software. At least three independent biological repeats were carried out for each effector.

\section{ACKNOWLEDGMENTS}

We thank S. Goldsmith for technical assistance and M. B. Mudgett for critical comments on the manuscript. This research was supported by grants from the Israel Science Foundation (ISF 326/10 to G. Sessa), the Deutsche Forschungsgemeinschaft (BR3875/2-2 to F. Brunner), and the COST Action Sustain (FA-1208) supported by COST (European Cooperation in Science and Technology) from the European Union.

\section{LITERATURE CITED}

Albert, M., Jehle, A. K., Mueller, K., Eisele, C., Lipschis, M., and Felix, G. 2010. Arabidopsis thaliana pattern recognition receptors for bacterial elongation factor Tu and flagellin can be combined to form functional chimeric receptors. J. Biol. Chem. 285:19035-19042.

Alfano, J. R., Charkowski, A. O., Deng, W. L., Badel, J. L., PetnickiOcwieja, T., van Dijk, K., and Collmer, A. 2000. The Pseudomonas syringae Hrp pathogenicity island has a tripartite mosaic structure composed of a cluster of type III secretion genes bounded by exchangeable effector and conserved effector loci that contribute to parasitic fitness and pathogenicity in plants. Proc. Natl. Acad. Sci. U.S.A. 97:4856-4861.

Asai, T., Tena, G., Plotnikova, J., Willmann, M. R., Chiu, W. L., GomezGomez, L., Boller, T., Ausubel, F. M., and Sheen, J. 2002. MAP kinase signalling cascade in Arabidopsis innate immunity. Nature 415:977-983.
Badel, J. L., Nomura, K., Bandyopadhyay, S., Shimizu, R., Collmer, A., and He, S. Y. 2003. Pseudomonas syringae pv. tomato DC3000 HopPtoM (CEL ORF3) is important for lesion formation but not growth in tomato and is secreted and translocated by the Hrp type III secretion system in a chaperone-dependent manner. Mol. Microbiol. 49:1239-1251.

Bartetzko, V., Sonnewald, S., Vogel, F., Hartner, K., Stadler, R., Hammes, U. Z., and Börnke, F. 2009. The Xanthomonas campestris pv. vesicatoria type III effector protein XopJ inhibits protein secretion: Evidence for interference with cell wall-associated defense responses. Mol. PlantMicrobe Interact 22:655-664.

Böhm, H., Albert, I., Fan, L., Reinhard, A., and Nürnberger, T. 2014. Immune receptor complexes at the plant cell surface. Curr. Opin. Plant Biol. 20:47-54.

Boller, T., and Felix, G. 2009. A renaissance of elicitors: Perception of microbe-associated molecular patterns and danger signals by patternrecognition receptors. Annu. Rev. Plant Biol. 60:379-406.

Canonne, J., Marino, D., Jauneau, A., Pouzet, C., Brière, C., Roby, D., and Rivas, S. 2011. The Xanthomonas type III effector XopD targets the Arabidopsis transcription factor MYB30 to suppress plant defense. Plant Cell 23:3498-3511.

Christmann, A., Hoffmann, T., Teplova, I., Grill, E., and Müller, A. 2005. Generation of active pools of abscisic acid revealed by in vivo imaging of water-stressed Arabidopsis. Plant Physiol. 137:209-219.

DebRoy, S., Thilmony, R., Kwack, Y.-B., Nomura, K., and He, S. Y. 2004. A family of conserved bacterial effectors inhibits salicylic acid-mediated basal immunity and promotes disease necrosis in plants. Proc. Natl. Acad. Sci. U.S.A. 101:9927-9932.

Felix, G., Duran, J. D., Volko, S., and Boller, T. 1999. Plants have a sensitive perception system for the most conserved domain of bacterial flagellin. Plant J. 18:265-276.

Feng, F., and Zhou, J. M. 2012. Plant-bacterial pathogen interactions mediated by type III effectors. Curr. Opin. Plant Biol. 15:469-476.

Fraiture, M., Zheng, X., and Brunner, F. 2014. An Arabidopsis and tomato mesophyll protoplast system for fast identification of early MAMPtriggered immunity-suppressing effectors. Methods Mol. Biol. 1127: 213-230.

Frederick, R. D., Thilmony, R. L., Sessa, G., and Martin, G. B. 1998. Recognition specificity for the bacterial avirulence protein AvrPto is determined by Thr-204 in the activation loop of the tomato Pto kinase. Mol. Cell 2:241-245.

Galán, J. E., Lara-Tejero, M., Marlovits, T. C., and Wagner, S. 2014. Bacterial type III secretion systems: Specialized nanomachines for protein delivery into target cells. Annu. Rev. Microbiol. 68:415-438.

Gimenez-Ibanez, S., Hann, D. R., Ntoukakis, V., Petutschnig, E., Lipka, V., and Rathjen, J. P. 2009. AvrPtoB targets the LysM receptor kinase CERK1 to promote bacterial virulence on plants. Curr. Biol. 19:423-429.

Göhre, V., Spallek, T., Häweker, H., Mersmann, S., Mentzel, T., Boller, T., de Torres, M., Mansfield, J. W., and Robatzek, S. 2008. Plant patternrecognition receptor FLS2 is directed for degradation by the bacterial ubiquitin ligase AvrPtoB. Curr. Biol. 18:1824-1832.

Gómez-Gómez, L., and Boller, T. 2000. FLS2: An LRR receptor-like kinase involved in the perception of the bacterial elicitor flagellin in Arabidopsis. Mol. Cell 5:1003-1011.

Goodin, M. M., Zaitlin, D., Naidu, R. A., and Lommel, S. A. 2008. Nicotiana benthamiana: Its history and future as a model for plantpathogen interactions. Mol. Plant-Microbe Interact 21:1015-1026.

Griesbeck, O., Baird, G. S., Campbell, R. E., Zacharias, D. A., and Tsien, R. Y. 2001. Reducing the environmental sensitivity of yellow fluorescent protein. Mechanism and applications. J. Biol. Chem. 276:29188-29194.

Guo, M., Tian, F., Wamboldt, Y., and Alfano, J. R. 2009. The majority of the type III effector inventory of Pseudomonas syringae pv. tomato DC3000 can suppress plant immunity. Mol. Plant-Microbe Interact 22:1069-1080.

He, P., Shan, L., Lin, N. C., Martin, G. B., Kemmerling, B., Nürnberger, T., and Sheen, J. 2006. Specific bacterial suppressors of MAMP signaling upstream of MAPKKK in Arabidopsis innate immunity. Cell 125:563-575.

Jamir, Y., Guo, M., Oh, H. S., Petnicki-Ocwieja, T., Chen, S., Tang, X., Dickman, M. B., Collmer, A., and Alfano, J. R. 2004. Identification of Pseudomonas syringae type III effectors that can suppress programmed cell death in plants and yeast. Plant J. 37:554-565.

Jones, J. B., Stall, R. E., and Bouzar, H. 1998. Diversity among xanthomonads pathogenic on pepper and tomato. Annu. Rev. Phytopathol. 36:41-58.

Jones, J. D. G., and Dangl, J. L. 2006. The plant immune system. Nature 444:323-329.

Kalogeraki, V. S., and Winans, S. C. 1997. Suicide plasmids containing promoterless reporter genes can simultaneously disrupt and create fusions to target genes of diverse bacteria. Gene 188:69-75.

Kim, J. G., Li, X., Roden, J. A., Taylor, K. W., Aakre, C. D., Su, B., Lalonde, S., Kirik, A., Chen, Y., Baranage, G., McLane, H., Martin, 
G. B., and Mudgett, M. B. 2009. Xanthomonas T3S effector XopN suppresses PAMP-triggered immunity and interacts with a tomato atypical receptor-like kinase and TFT1. Plant Cell 21:1305-1323.

Kim, J. G., Stork, W., and Mudgett, M. B. 2013. Xanthomonas type III effector XopD desumoylates tomato transcription factor SIERF4 to suppress ethylene responses and promote pathogen growth. Cell Host Microbe 13:143-154.

Kim, J. G., Taylor, K. W., Hotson, A., Keegan, M., Schmelz, E. A., and Mudgett, M. B. 2008. XopD SUMO protease affects host transcription, promotes pathogen growth, and delays symptom development in Xanthomonas-infected tomato leaves. Plant Cell 20:1915-1929.

Kim, M. G., and Mackey, D. 2008. Measuring cell-wall-based defenses and their effect on bacterial growth in Arabidopsis. Methods Mol. Biol. 415: 443-452.

Kovach, M. E., Elzer, P. H., Hill, D. S., Robertson, G. T., Farris, M. A., Roop, R. M., 2nd, and Peterson, K. M. 1995. Four new derivatives of the broad-host-range cloning vector pBBR1MCS, carrying different antibiotic-resistance cassettes. Gene 166:175-176.

Kruse, T., Gehl, C., Geisler, M., Lehrke, M., Ringel, P., Hallier, S., Hänsch, R., and Mendel, R. R. 2010. Identification and biochemical characterization of molybdenum cofactor-binding proteins from Arabidopsis thaliana. J. Biol. Chem. 285:6623-6635.

Kunze, G., Zipfel, C., Robatzek, S., Niehaus, K., Boller, T., and Felix, G. 2004. The $\mathrm{N}$ terminus of bacterial elongation factor $\mathrm{Tu}$ elicits innate immunity in Arabidopsis plants. Plant Cell 16:3496-3507.

Lin, W., Ma, X., Shan, L., and He, P. 2013. Big roles of small kinases: The complex functions of receptor-like cytoplasmic kinases in plant immunity and development. J. Integr. Plant Biol. 55:1188-1197.

Macho, A. P., and Zipfel, C. 2014. Plant PRRs and the activation of innate immune signaling. Mol. Cell 54:263-272.

Macho, A. P., and Zipfel, C. 2015. Targeting of plant pattern recognition receptor-triggered immunity by bacterial type-III secretion system effectors. Curr. Opin. Microbiol. 23:14-22.

Minsavage, G. V., Dahlbeck, D., Whalen, M. C., Kearney, B., Bonas, U., Staskawicz, B. J., and Stall, R. E. 1990. Gene-for-gene relationships specifying disease resistance in Xanthomonas campestris pv. vesicatoriapepper interactions. Mol. Plant-Microbe Interact 3:41-47.

Nelson, B. K., Cai, X., and Nebenführ, A. 2007. A multicolored set of in vivo organelle markers for co-localization studies in Arabidopsis and other plants. Plant J. 51:1126-1136.

Nguyen, H. P., Chakravarthy, S., Velásquez, A. C., McLane, H. L., Zeng, L., Nakayashiki, H., Park, D. H., Collmer, A., and Martin, G. B. 2010. Methods to study PAMP-triggered immunity using tomato and Nicotiana benthamiana. Mol. Plant-Microbe Interact 23:991-999.

Roden, J. A., Belt, B., Ross, J. B., Tachibana, T., Vargas, J., and Mudgett, M. B. 2004. A genetic screen to isolate type III effectors translocated into pepper cells during Xanthomonas infection. Proc. Natl. Acad. Sci. U.S.A. 101:16624-16629.

Rott, P. C., Costet, L., Davis, M. J., Frutos, R., and Gabriel, D. W. 1996. At least two separate gene clusters are involved in albicidin production by Xanthomonas albilineans. J. Bacteriol. 178:4590-4596.

Schulze, S., Kay, S., Büttner, D., Egler, M., Eschen-Lippold, L., Hause, G., Krüger, A., Lee, J., Müller, O., Scheel, D., Szczesny, R., Thieme, F., and Bonas, U. 2012. Analysis of new type III effectors from Xanthomonas uncovers XopB and XopS as suppressors of plant immunity. New Phytol. 195:894-911.

Scott, J. W., Jones, J. B., Somodi, G. C., and Stall, R. E. 1995. Screening tomato accessions for resistance to Xanthomonas campestris pv. vesicatoria, race T3. HortScience 30:579-581.

Shan, L., He, P., Li, J., Heese, A., Peck, S. C., Nürnberger, T., Martin, G. B., and Sheen, J. 2008. Bacterial effectors target the common signaling partner BAK1 to disrupt multiple MAMP receptorsignaling complexes and impede plant immunity. Cell Host Microbe 4:17-27.

Singer, A. U., Schulze, S., Skarina, T., Xu, X., Cui, H., Eschen-Lippold, L., Egler, M., Srikumar, T., Raught, B., Lee, J., Scheel, D., Savchenko, A., and Bonas, U. 2013. A pathogen type III effector with a novel E3 ubiquitin ligase architecture. PLoS Pathog. 9:e1003121.
Stork, W., Kim, J. G., and Mudgett, M. B. 2015. Functional analysis of plant defense suppression and activation by the Xanthomonas core type III effector XopX. Mol. Plant-Microbe Interact 28:180-194.

Szczesny, R., Büttner, D., Escolar, L., Schulze, S., Seiferth, A., and Bonas, U. 2010. Suppression of the AvrBs1-specific hypersensitive response by the YopJ effector homolog AvrBsT from Xanthomonas depends on a SNF1-related kinase. New Phytol. 187:1058-1074.

Teper, D., Burstein, D., Salomon, D., Gershovitz, M., Pupko, T., and Sessa, G. 2016. Identification of novel Xanthomonas euvesicatoria type III effector proteins by a machine-learning approach. Mol. Plant Pathol. 17: 398-411.

Teper, D., Salomon, D., Sunitha, S., Kim, J. G., Mudgett, M. B., and Sessa, G. 2014. Xanthomonas euvesicatoria type III effector XopQ interacts with tomato and pepper 14-3-3 isoforms to suppress effector-triggered immunity. Plant J. 77:297-309.

Thieme, F., Koebnik, R., Bekel, T., Berger, C., Boch, J., Büttner, D., Caldana, C., Gaigalat, L., Goesmann, A., Kay, S., Kirchner, O., Lanz, C., Linke, B., McHardy, A. C., Meyer, F., Mittenhuber, G., Nies, D. H., Niesbach-Klösgen, U., Patschkowski, T., Rückert, C., Rupp, O., Schneiker, S., Schuster, S. C., Vorhölter, F. J., Weber, E., Pühler, A., Bonas, U., Bartels, D., and Kaiser, O. 2005. Insights into genome plasticity and pathogenicity of the plant pathogenic bacterium Xanthomonas campestris pv. vesicatoria revealed by the complete genome sequence. J. Bacteriol. 187:7254-7266.

Thieme, F., Szczesny, R., Urban, A., Kirchner, O., Hause, G., and Bonas, U. 2007. New type III effectors from Xanthomonas campestris pv vesicatoria trigger plant reactions dependent on a conserved $\mathrm{N}$-myristoylation motif. Mol. Plant-Microbe Interact 20:1250-1261.

Üstün, S., Bartetzko, V., and Börnke, F. 2013. The Xanthomonas campestris type III effector XopJ targets the host cell proteasome to suppress salicylic-acid mediated plant defence. PLoS Pathog. 9:e1003427.

Xiang, T., Zong, N., Zhang, J., Chen, J., Chen, M., and Zhou, J. M. 2011. BAK1 is not a target of the Pseudomonas syringae effector AvrPto. Mol. Plant-Microbe Interact 24:100-107.

Xiang, T., Zong, N., Zou, Y., Wu, Y., Zhang, J., Xing, W., Li, Y., Tang, X., Zhu, L., Chai, J., and Zhou, J. M. 2008. Pseudomonas syringae effector AvrPto blocks innate immunity by targeting receptor kinases. Curr. Biol. 18:74-80.

Yoo, S. D., Cho, Y. H., and Sheen, J. 2007. Arabidopsis mesophyll protoplasts: A versatile cell system for transient gene expression analysis. Nat. Protoc. 2:1565-1572.

Zeng, L., Velásquez, A. C., Munkvold, K. R., Zhang, J., and Martin, G. B. 2012. A tomato LysM receptor-like kinase promotes immunity and its kinase activity is inhibited by AvrPtoB. Plant J. 69:92-103.

Zhang, J., Li, W., Xiang, T., Liu, Z., Laluk, K., Ding, X., Zou, Y., Gao, M., Zhang, X., Chen, S., Mengiste, T., Zhang, Y., and Zhou, J. M. 2010. Receptor-like cytoplasmic kinases integrate signaling from multiple plant immune receptors and are targeted by a Pseudomonas syringae effector. Cell Host Microbe 7:290-301.

Zhang, J., Shao, F., Li, Y., Cui, H., Chen, L., Li, H., Zou, Y., Long, C., Lan, L., Chai, J., Chen, S., Tang, X., and Zhou, J. M. 2007. A Pseudomonas syringae effector inactivates MAPKs to suppress PAMP-induced immunity in plants. Cell Host Microbe 1:175-185.

Zhang, J., and Zhou, J. M. 2010. Plant immunity triggered by microbial molecular signatures. Mol. Plant 3:783-793.

Zheng, X., McLellan, H., Fraiture, M., Liu, X., Boevink, P. C., Gilroy, E. M., Chen, Y., Kandel, K., Sessa, G., Birch, P. R., and Brunner, F. 2014. Functionally redundant RXLR effectors from Phytophthora infestans act at different steps to suppress early flg22-triggered immunity. PLoS Pathog. 10:e1004057.

Zuo, J., Niu, Q. W., and Chua, N. H. 2000. Technical advance: An estrogen receptor-based transactivator XVE mediates highly inducible gene expression in transgenic plants. Plant J. 24:265-273.

\section{AUTHOR-RECOMMENDED INTERNET RESOURCE}

Icy software platform: icy.bioimageanalysis.org 